with an inappropriately elevated insulin $(14.3 \mathrm{mU} / \mathrm{l})$ and cpeptide (1063 pmol/l). Gastrin was raised $(55 \mathrm{pmol} / \mathrm{l})$. CT, MRCP, MRI pancreas and EUS were unremarkable. DOTATATE showed a small lesion in the pancreatic tail. Surgical enucleation of the lesion revealed a well differentiated NET histologically.

Conclusion These cases support the use of Ga-DOTATATE as a potential diagnostic tool in suspected but not yet localised primary cases of gastrinoma/insulinoma with symptoms or elevated blood levels of tumour markers where cross-sectional imaging is normal or equivocal.

To date calcium stimulation with selective angiography has been used. Ga-DOTATATE may obviate the need for this invasive and highly complex test.

Larger case series and prospective data are needed to look at the validity of this test and ascertain its role in routine clinical practice.

Disclosure of Interest None Declared.

\section{PTH-099 EXTERNAL SHOCKWAVE LITHOTRIPSY (ESWL) OF PANCREATIC CALCULI IMPROVES PAIN RELATED TO CHRONIC CALCIFIC PANCREATITIS}

${ }^{1} \mathrm{~K}$ Kandiah*, ${ }^{2} \mathrm{SF}$ Neong, ${ }^{2} \mathrm{P}$ Vlavianos, ${ }^{1} \mathrm{D}$ Bansi, ${ }^{1} \mathrm{M}$ Hanna, ${ }^{2} \mathrm{D}$ Westaby. ${ }^{1}$ Gastroenterology, Charing Cross Hospital, Imperial Healthcare NHS Trust, London, UK; ${ }^{2}$ Gastroenterology, Hammersmith Hospital, Imperial Healthcare NHS Trust, London, UK

\subsection{6/gutjnl-2014-307263.545}

Introduction Chronic calcific pancreatitis is associated with the development of pancreatic ductal calculi. The calculi can lead to the blockage of the pancreatic duct which can increase the pressure in the duct causing pain. Removal of pancreatic calculi is conventionally done using endoscopic retrograde cholangio-pancreatiocography (ERCP). However, removal of large pancreatic calculi may not be amenable using ERCP alone. External shockwave lithotripsy has been successfully used to target and fragment large calculi located in the head or body of the pancreas. The fragmented calculi can be extracted by subsequent ERCP.

Methods We conducted a retrospective case-control study. We identified a cohort of patients who underwent ESWL followed by ERCP for the clearance of large calculi in the pancreatic duct and a cohort who were treated conventionally with ERCP +/pancreatic duct stenting over a 15 -month period from 22 August 2012 to 21 November 2013 in a tertiary hepatopancreatobiliary centre. The medical notes, endoscopy reports and radiological imaging of these patients were reviewed retrospectively to assess the success of achieving ductal clearance and the improvement in abdominal pain.

Results We identified 9 patients who underwent ESWL followed by ERCP and a same number of matched controls. Complete ductal clearance following ESWL/ERCP was 6 (66.7\%) and partial in $3(33.3 \%)$. 1 patient required 2 sessions of ESWL. Following ESWL/ERCP, 4 (44\%) patients had no pain, 4(44\%) had mild to moderate pain and 1 had severe pain. In the control group, 2 had no pain, 2 had mild to moderate pain and the rest still experienced severe pain. There were no complications following ESWL.

Conclusion ESWL combined with ERCP is safe and efficient in providing symptomatic relief for patients with large pancreatic calculi related to chronic pancreatitis. It can be offered as first line therapy in select patients with large pancreatic calculi.

\section{REFERENCES}

1 Tandan M, Reddy DN, Santosh D, Reddy V, Koppuju V, Lakhtakia S, Gupta R, Ramchandani M, Rao GV. Extracorporeal shock wave lithotripsy of large difficult common bile duct stones: efficacy and analysis of factors that favor stone fragmentation. J Gastroenterol Hepatol 2009:24:1370-1374

2 Ellis RD, Jenkins AP, Thompson RP, Ede RJ. Clearance of refractory bile duct stones with extracorporeal shockwave lithotripsy. Gut 2000:47:728-731

3 Lawrence C, Siddiqi MF, Hamilton JN, Keane TE, Romagnuolo J, Hawes RH, Cotton PB. Chronic calcific pancreatitis: combination ERCP and extracorporeal shock wave lithotripsy for pancreatic duct stones. South Med J 2010 Jun;103(6):505-8

Disclosure of Interest None Declared.

\section{PTH-100 UTILITY OF EUS-GUIDED FINE NEEDLE ASPIRATION OF PANCREATIC CYSTIC LESIONS}

MC Donnelly*, P Cheng, JS Leeds. Gastroenterology, NHS Grampian, Aberdeen, UK

\subsection{6/gutjnl-2014-307263.546}

Introduction At least 1\% of hospitalised patients will have a pancreatic cystic lesion on cross sectional imaging ${ }^{1}$. Differentiation of benign and potentially malignant/malignant pancreatic cystic lesions using conventional radiology and prior to surgery is difficult. Endoscopic ultrasound (EUS) guided fine needle aspiration (FNA) is considered safe, yet there is relatively limited data on the clinical utility of EUS in this setting or its complication rate.

Methods Retrospectively, 43 consecutive patients ( $\mathrm{F}=26$, $\mathrm{M}=17$, mean age 63) undergoing EUS-FNA of a pancreatic cystic lesion (no. procedures $=46$ ) following abnormal imaging were identified from an institutionally approved database. Data collected included pre-EUS imaging, EUS findings, number of passes, cyst fluid CEA, amylase and cytology, sedation requirements, complication rate and six month follow up where available.

Results 44 procedures provided sufficient information for further analysis. At EUS, 23 cystic lesions appeared benign and 21 premalignant/malignant. Median CEA (ug/L) in the benign group was 6 vs. 2234 in the malignant group; p $<0.001$. Median amylase $(\mathrm{U} / \mathrm{L})$ in the benign group was 2989 , versus 2795 in the malignant group; $\mathrm{p}=1.0$. In the malignant group, $4 / 21(19 \%)$ had positive cytology. In the benign group, 16/23 (69.5\%) had no malignant cells.

Cytology was insufficient for analysis in $4 / 23$ of benign appearing lesions, and 3/21 in malignant appearing lesions.

Average midazolam dose was $3.3 \mathrm{mg}$, and average pethidine dose $27.9 \mathrm{mg}$.

Complication rate was $4 \%(n=2)$, with one patient experiencing severe abdominal pain (serum amylase normal) and another having a documented bile leak.

Availability of follow up data was limited by patients returning to their secondary care referral centres. 4 patients proceeded to surgery and had resection pathology available. Of these, 3 patients had confirmed malignant IPMN (one patient had no cyst fluid for analysis, one had raised CEA and no available

\begin{tabular}{lll} 
Abstract PTH-100 Table $\mathbf{1}$ & & \\
\hline & CEA ug/L & Cytology \\
\hline Sensitivity & 80 & 18.2 \\
Specificity & 100 & 100 \\
PPV & 100 & 100 \\
NPV & 83 & 55 \\
\hline
\end{tabular}


cytology, and one had suggestive cytology but no CEA analysis). The fourth patient had a mucinous cystadenoma, with mucin aspirated at FNA but no CEA analysed.

Conclusion EUS provides useful information with regards to the macroscopic appearances of pancreatic cystic lesions. FNA is safe and allows the addition of fluid CEA as a useful marker for malignancy. Negative cytology does not exclude malignancy but false positives are rare. Difficulty in aspirating fluid should be considered suspicious.

\section{REFERENCE}

1 Khalid A, Brugge W. ACG Practice guidelines for the diagnosis and management of neoplastic pancreatic cysts. Am Journ Gastroenterol (2007);102:2339-2349

Disclosure of Interest None Declared.

\section{PTH-101 DIETARY SATURATED FATTY ACIDS ARE POSITIVELY ASSOCIATED WITH THE DEVELOPMENT OF PANCREATIC CANCER - DATA FROM A UK PROSPECTIVE STUDY (EPIC) USING 7-DAY FOOD DIARIES}

${ }^{1} \mathrm{P}$ Banim*, ${ }^{2} \mathrm{R}$ Luben, ${ }^{2} \mathrm{~K}$-T Khaw, ${ }^{3} \mathrm{~N}$ Wareham, ${ }^{4} \mathrm{~A}$ Hart. ${ }^{1}$ Gastroenterology, James Paget University Hospital, Great Yarmouth, UK; ${ }^{2}$ Department of Public Health, Institute of Public Health, University of Cambridge, Cambridge, UK; ${ }^{3}$ MRC Epidemilogy Unit, University of Cambridge, Cambridge, UK; ${ }^{4}$ Norwich Medical School, University of East Anglia, Norwich, UK

\subsection{6/gutjnl-2014-307263.547}

Introduction An increased dietary intake of saturated fatty acids could promote pancreatic carcinogenesis through increasing insulin resistance. Our aim was to investigate this association for the first time using nutrient information measured by food diaries in a cohort study.

Methods A total of 23,658 men and women aged 40-74 years recruited between 1993-1997 into the EPIC-Norfolk study, UK, completed 7-day food diaries which recorded all foods, brands, recipes and portion sizes eaten. The diaries were interpreted by nutritionists using a computer programme which converted participant recorded text into nutrient values of: total saturated fatty acids intake, palmitic and stearic acids. The cohort was monitored until June 2010 to identify those participants who developed incident pancreatic cancer, confirmed by a review of medical notes. The HRs of developing cancer were estimated across quintiles of intake using Cox regression, adjusted for total energy intake and oleic acid intake, the latter which increases insulin sensitivity.

Results During 17 years of follow-up, 88 participants were diagnosed with pancreatic cancer $(55 \%$ women, median age at diagnosis $=73.4$ years, range $52.1-88.8$ years). There were no associations with either total or individual saturated fatty acids in the whole cohort or in women. However, in men, increasing quintiles of dietary saturated fat acids were positively associated with risk (highest vs. lowest quintile HR $=9.68$, 95\% CI: 1.72 54.54, $\mathrm{P}=0.01$ ) with a trend across quintiles (Trend $\mathrm{HR}=1.76,95 \%$ CI: 1.17-2.64). There were similar associations for palmitic acid (trend HR $=2.29,95 \% \mathrm{CI}$ : 1.41.3.71) and stearic acid (trend $\mathrm{HR}=1.51,95 \% \mathrm{CI}: 1.00-2.30$ ).

Conclusion The data support an aetiological role of dietary saturated fatty acids in pancreatic cancer in men, due to the large effect sizes, dose-responses, temporality and plausible biological mechanisms. These macronutrients should be measured in future aetiological studies and the reasons for differences between genders investigated.

Disclosure of Interest None Declared.

\section{PTH-102 TREATMENT OF BENIGN PANCREATIC DUCT STRICTURES WITH FULLY COVERED SELF EXPANDABLE METAL STENTS (FC-SEMS) IS FEASIBLE, SAFE AND EFFECTIVE IN PATIENTS WITH SYMPTOMATIC CHRONIC PANCREATITIS (CP)}

*R Rameshshanker, H Kaltsidis, P Vlavianos, N Phillips, D Westaby. Gastroenterology, Imperial College Health Care NHS Trust: Hammersmith Hospital, London, UK

\subsection{6/gutjnl-2014-307263.548}

Introduction FC-SEMS have been used for treatment of benigh biliary strictures (BBS) with excellent results. Pancreatic duct strictures (PDS) of CP vary in length and complexity and associated with upstream dilatation and/or stones. Limited data on aggressive endotherapy (ET) with insertion of the repeated plastic stents (PS) showed good response. Experience with FC-SEMS in pancreatic strictures is limited.

Methods Aim:To assess feasibility and safety of smaller diameter $(8 \mathrm{~mm})$ FC-SEMS for treatment of CP with dominant downstream strictures either de novo or as resque (failed previous ET).

\begin{tabular}{|c|c|c|c|c|c|c|c|c|}
\hline Patients & Age & Sex & De Novo $(\mathrm{N})$ or rescue $(\mathrm{R})$ & Main (M) or Santorini (S) & ESWL & $\begin{array}{l}\text { Pain response } \\
\text { partial }(\mathrm{P}) \text {, complete (C);nil (N) }\end{array}$ & Complications & SEMS removed in weeks \\
\hline 1 & 43 & M & $\mathrm{N}$ & M & - & C & None & 13 \\
\hline 2 & 67 & M & $\mathrm{R}$ & M & - & $P$ & None & 12 \\
\hline 3 & 66 & $\mathrm{~F}$ & $\mathrm{R}$ & $S$ & - & $P$ & None & 11 \\
\hline 4 & 71 & $\mathrm{~F}$ & $\mathrm{R}$ & M & + & C & None & 12 \\
\hline 5 & 32 & M & $\mathrm{R}$ & M & + & C & None & 16 \\
\hline 6 & 55 & $\mathrm{~F}$ & N & S & - & C & None & 12 \\
\hline 7 & 68 & $\mathrm{~F}$ & $\mathrm{R}$ & S & - & C & None & 16 \\
\hline 8 & 47 & $\mathrm{~F}$ & $\mathrm{R}$ & M & - & $\mathrm{N}$ & None & 04 \\
\hline 9 & 40 & M & $\mathrm{R}$ & M & - & C & None & 10 \\
\hline 10 & 59 & M & $\mathrm{R}$ & M & + & C & None & 12 \\
\hline 11 & 55 & $F$ & $\mathrm{R}$ & $S$ & - & $\mathrm{N}$ & None & 12 \\
\hline 12 & 47 & M & $\mathrm{R}$ & M & + & $\mathrm{N}$ & None & 12 \\
\hline 13 & 36 & $\mathrm{~F}$ & $\mathrm{R}$ & M & - & C & None & 20 \\
\hline 14 & 41 & $\mathrm{~F}$ & $\mathrm{R}$ & $S$ & - & C & None & 12 \\
\hline 15 & 57 & $\mathrm{~F}$ & $\mathrm{R}$ & M & - & $\mathrm{N}$ & proximal migration+ Santorini abscess & 02 \\
\hline
\end{tabular}

